\title{
Genotype distribution characteristics of high-risk human papillomaviruses in women from Shanghai, China
}

\author{
Y. GU, M. YI, Y. XU, H. ZHAO, F. FU AND Y. ZHANG* \\ Department of Gynaecology and Obstetrics, the Shanghai 7th People's Hospital, 358 Datong Road, the New \\ Pudong District, Shanghai, China
}

Received 26 August 2015; Final revision 6 October 2015; Accepted 14 October 2015; first published online 11 November 2015

\section{SUMMARY}

High-risk human papillomaviruses (HPVs) are highly prevalent worldwide, and HPV genotype distribution varies regionally. Molecular surveys of HPVs are important for effective HPV control and prevention. Fifteen high-risk HPV strains (16, 18, 31, 33, 35, 39, 45, 51, 52, 53, 56, 58, 59, 66, 68) and six low-risk HPV strains (HPV6, 11, 42, 43, 44, CP8304) were detected by cervical cytology from 10501 subjects. High-risk HPVs, low-risk HPVs, and both high- and low-risk HPVs were detected in $14 \cdot 5 \%, 2 \cdot 8 \%$, and $2 \cdot 4 \%$ of cases, respectively. Of 1782 subjects with high-risk HPV infection, $75 \cdot 5 \%, 18 \cdot 1 \%$, and $6 \cdot 4 \%$ were infected with one, two, and $\geqslant 3$ strains of high-risk HPVs, respectively. HPV52, HPV16, and HPV58 were the top three most dominant high-risk HPV genotypes in our population with positivity rates of $23 \cdot 0 \%, 17 \cdot 7 \%$ and $16.9 \%$, respectively. Multiple infection was common, with significantly higher co-infection rates of HPV58/HPV33 (12.9\%) and HPV58/HPV52 (11.3\%). Further data comparisons showed that HPV genotype distribution varied markedly between domestic and international regions. In conclusion, a monolithic vaccination strategy is obviously impractical, and regional HPV surveillance is essential to optimize current HPV control and prevention.

Key words: Human papilloma virus (HPV), molecular survey, viral strain distribution.

\section{INTRODUCTION}

Approximately $10-15 \%$ of human cancers are caused by infection with high-risk human papillomaviruses (HPVs), Epstein-Barr virus, hepatitis B virus, human T-lymphotropic virus-I, hepatitis C virus, Kaposi's sarcoma herpesvirus, and Merkel cell polyomavirus [1]. HPV is a double-stranded DNA virus belonging to the papillomavirus family that is capable of infecting the basal cells of the stratified epithelium in humans [2]. HPVs are highly prevalent worldwide,

\footnotetext{
* Author for correspondence: Dr Y. Zhang, Department of Gynaecology and Obstetrics, the Shanghai 7th People's Hospital, 358 Datong Road, the New Pudong District, Shanghai 200137, China.

(Email: zyzhangyu2015@sina.com)
}

and high-risk HPV infections cause various cancers, including vaginal, vulvar, penile, oropharyngeal, anal, and cervical [3-6]. Cervical cancer, which is mainly categorized into squamous cell carcinoma and adenocarcinoma, occurs when abnormal cells of the cervix grow out of control, and is a leading cause of death in women with 0.5 million new cases and 275000 deaths worldwide each year [7]. Significantly, almost all cases of cervical cancer are caused by long-lasting infections with certain types of human HPV [8].

The genome of HPVs consists of a circular DNA molecule of $\sim 8000$ base pairs subdivided into the early (E) region, upstream regulatory region (URR), and late $(\mathrm{L})$ region $[9,10]$. The $\mathrm{E}$ region encodes at least eight early viral proteins: E1, responsible for 
viral DNA replication; E2, responsible for activating the replication of viral DNA in synergy with E1 and transcriptional repression of E6 and E7 in the nucleus, and responsible for induction of apoptosis and genomic instability in the cytoplasm; E4, responsible for virion maturation; E5, responsible for stimulation of cell proliferation; E6, responsible for oncogenic protein promoting degradation of $\mathrm{p} 53$ by the proteasome; E7, responsible for oncogenic protein promoting degradation of retinoblastoma susceptibility protein; and E3 and E8, of no known functions [9, 10]. The L region encodes the major protein capsid protein L1 and the minor protein L2 to form the viral particle $[9,10]$. To date, over $170 \mathrm{HPV}$ genotypes have been identified [11], with types $16,18,31,33,35,39,45$, $51,52,56,58,59,68,73$, and 82 defined as high-risk, carcinogenic, sexually transmitted HPVs that may lead to the development of cervical cancer [12]. The diagnosis of HPV infection is based primarily on detection of viral DNA $[12,13]$. Genotyping of HPV is mainly based on the unique sequence in the L1 region of the HPV genome [14]. HPV genotypes are not only disease-specific but also strain-specific within a certain disease or cancer [15-18]. HPV6, 11, 42, and 44 infections are correlated with anogenital warts [15]. HPV16 infection causes oropharyngeal cancer [16]. HPV16 and 18 are most prevalent in head and neck squamous cell carcinomas [17]. HPV16, 18, 26, 31, $33,35,39,45,51,52,53,56,58,59,66,68,73$, and 82 infections cause genital cancers [12, 18].

As HPVs are highly prevalent worldwide and genotypes differ among regions, molecular epidemiological survey of HPVs could provide background data regarding characteristic HPV genotype distribution and composition in a certain area and therefore support local vaccination strategies. For this purpose, the distribution characteristics of high-risk HPV genotypes were studied in women living in Shanghai.

\section{METHODS}

\section{Study population}

The Shanghai 7th Hospital is a general hospital located in northeastern Shanghai. Outpatients visiting our Department of Gynecology between January 2014 and May 2015 for cervical screening were included in this study. All participants were screened by clinical gynecological examination, cytology examination, and colposcopy examination. Age, clinical data, and HPV testing results were recorded. Inclusion criteria were a sexual history, not currently pregnant, no history of total uterus or cervix resection, and agreement to undergo HPV testing. Patients that had visited a doctor 2 months previously and received cervical physical therapy and hormone treatment were excluded from the study. A total of 10501 subjects (age: median 48; quartile range 39-55 years) were included. For Pap smears, cells were collected at the outer opening of the cervix at the transformation zone and then examined under a microscope to detect any abnormalities in the exfoliated cervical cells. Pap smears were interpreted by experienced pathologists in our hospital. Cytological diagnoses were evaluated according to the Bethesda System criteria [19]. At the same time, the cell samples were analysed to detect HPV DNA.

\section{Ethical standards}

This study was conducted in accordance with the World Medical Association Declaration of Helsinki. The Review Board of the Ethics Committee of Medical Research at Shanghai Seventh People's Hospital (Shanghai, China) approved the study protocols. Written informed consent was obtained from all patients according to the guidelines of the Chinese National Ethics Regulation Committee. All patients were informed of their rights to withdraw consent personally or via relatives, caregivers, or guardians.

The authors assert that all procedures contributing to this work comply with the ethical standards of the relevant national and institutional committees on human experimentation and with the Helsinki Declaration of 1975, as revised in 2008.

\section{HPV genotyping}

HPV genotyping was performed using a commercial detection kit purchased from Hybribio (Chaozhou, China). The kit could detect and distinguish 15 highrisk HPV strains (HPV16, 18, 31, 33, 35, 39, 45, 51, $52,53,56,58,59,66,68)$ and six low-risk HPV strains (HPV6, 11, 42, 43, 44, CP8304) (81). The kit is based on polymerase chain reaction followed by HPV DNA microarray analyses. All detection procedures were guided by the protocol provided by manufacturers. Detected HPV strains were defined by genotype not by sub-genotype or quasi-species [20]. 

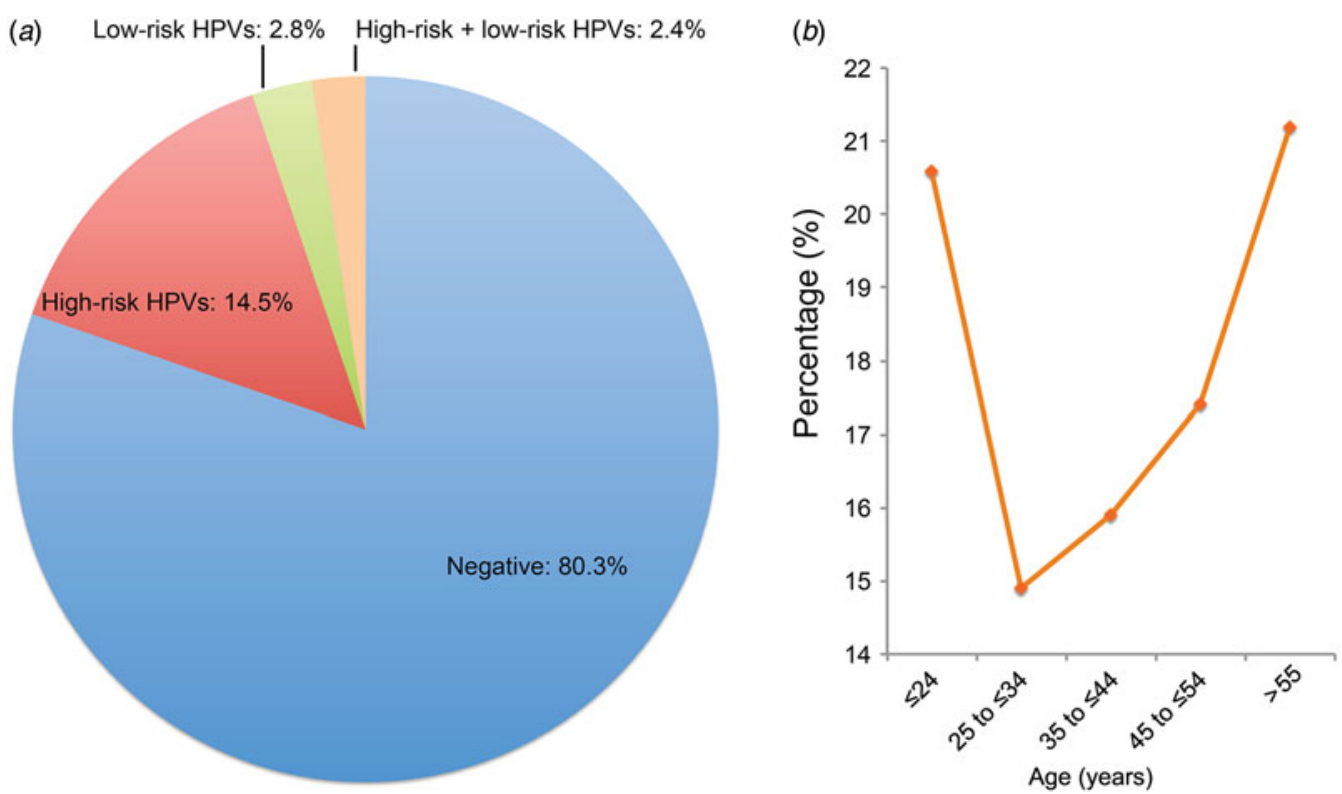

Fig. 1. Overall prevalence of human papillomavirus (HPV) and age-specific distribution. (a) Overall prevalence of high-risk and low-risk HPVs in 10501 subjects. (b) Distribution characteristics of high-risk and low-risk HPVs according to age stratification for 10501 subjects.

\section{Statistical analysis}

Statistical analysis was performed using SPSS v. 13.0 (SPSS Inc., USA). Continuous variables are presented as means \pm standard deviation (s.D.) and categorical data are presented as numbers (percentages). Differences between groups were examined using the $t$ test, one-way ANOVA, $c^{2}$ test, or Fisher's exact probability test according to the characteristics of data distribution. The significance level $\alpha$ was set at $0 \cdot 05$.

\section{RESULTS}

\section{Overall prevalence of HPV}

Data from a total of 10501 outpatients were collected; $8430(80 \cdot 3 \%)$ were negative for HPV. The overall prevalence of HPV was 19.7\% (2071 patients), $14.5 \%$ (1525 patients) of whom were infected with high-risk HPVs, 2.8\% (289 patients) were infected with low-risk HPVs, and 2.4\% (257 patients) were co-infected with both (Fig. 1a). The overall HPV prevalence rates were calculated for the following age groups: $\leqslant 24$ years, $20.6 \%$; $25-34$ years, $14.9 \%$; $35-44$ years, $15 \cdot 9 \%$; 45-55 years, $17 \cdot 4 \%$; and $>55$ years, $21 \cdot 2 \%$ (Fig. 1b). The prevalence ratio of HPV infection decreased from $20.6 \%$ in subjects aged $<24$ years to the lowest ratio of $14.9 \%$ in those aged $25-34$ years, and then increased to $21 \cdot 2 \%$ in subjects aged $>55$ years (Fig. $1 b$ ).

\section{Prevalence characteristic of high-risk HPVs}

A total of 1782 subjects were infected with high-risk HPVs. Of these, 1346 (75.5\%) subjects were infected with a single high-risk HPV strain, $322(18 \cdot 1 \%)$ subjects were infected with two high-risk strains, and $114(6 \cdot 4 \%)$ subjects were infected with $\geqslant 3$ high-risk strains (Fig. 2a). The average ages of subjects infected with one, two, and $\geqslant 3 \mathrm{HPV}$ strains were $39 \cdot 5 \pm 12 \cdot 4$, $40 \cdot 2 \pm 13 \cdot 6$, and $36 \cdot 6 \pm 15 \cdot 8$ years, respectively (Fig. 2b). The average age of subjects infected with $\geqslant 3$ HPV strains was significantly younger than that of the other two groups (Fig. 2b).

\section{Distribution characteristics of high-risk HPV genotypes}

As shown in Table 1, HPV genotypes 16, 18, 31, 33, $35,39,45,51,52,53,56,58,59,66$, and 68 were detected in $17 \cdot 7 \%, 6 \cdot 3 \%, 7 \cdot 5 \%, 10 \cdot 4 \%, 2 \cdot 2 \%, 9 \cdot 3 \%$, $2 \cdot 1 \%, 9 \cdot 3 \%, 23 \cdot 0 \%, 10 \cdot 8 \%, 3 \cdot 1 \%, 16 \cdot 9 \%, 2 \cdot 7 \%, 4 \cdot 9 \%$, and $7 \cdot 0 \%$ of the 1782 high-risk HPV-infected subjects, respectively. The top three most dominant HPV genotypes prevalent in our population were HPV52, HPV16, and HPV58.

\section{Characteristics of HPV co-infection}

As described above, multiple HPV infection was common in this population. To determine the co-infection 

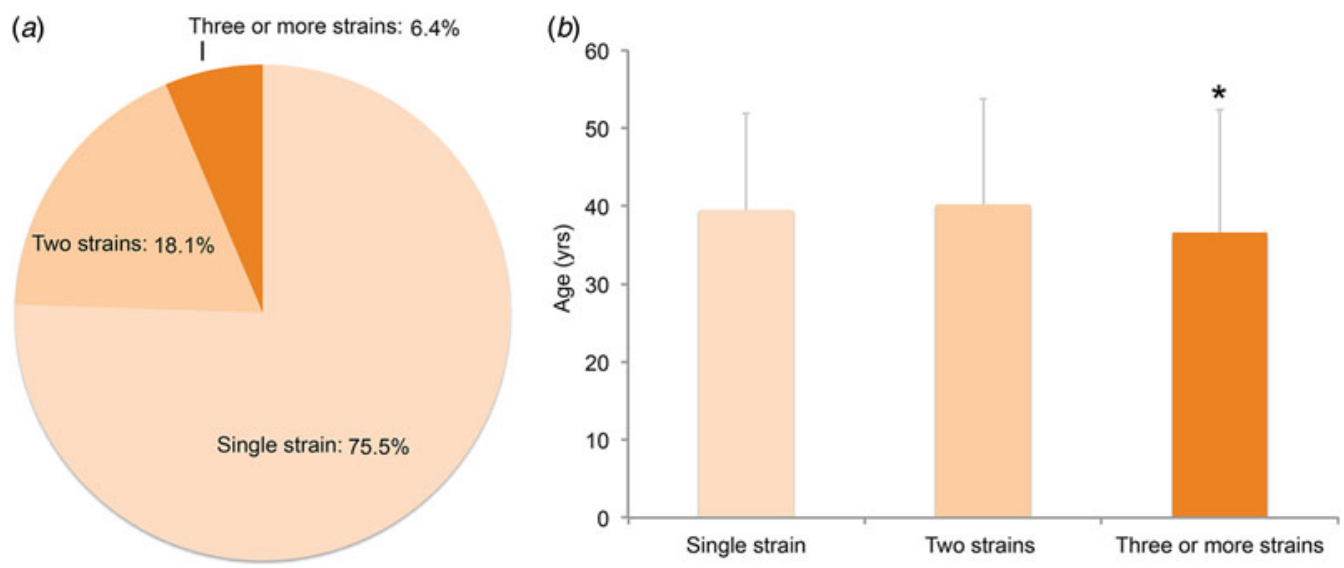

Fig. 2. Prevalence and distribution characteristics of high-risk human papillomaviruses (HPVs). (a) Constituent ratios of single and multiple infection in 1782 high-risk HPV-infected subjects. (b) Average ages of subject groups infected by one, two, and $\geqslant 3 \mathrm{HPV}$ strains. $* P<0.05$ compared to the other two groups.

Table 1. Distribution characteristics of high-risk HPV genotypes $(n=1782)$

\begin{tabular}{llr}
\hline \hline Genotype & Number & \multicolumn{1}{c}{$(\%)$} \\
\hline HPV16 & 316 & $(17 \cdot 7)$ \\
HPV18 & 112 & $(6 \cdot 3)$ \\
HPV31 & 134 & $(7 \cdot 5)$ \\
HPV33 & 185 & $(10 \cdot 4)$ \\
HPV35 & 39 & $(2 \cdot 2)$ \\
HPV39 & 165 & $(9 \cdot 3)$ \\
HPV45 & 37 & $(2 \cdot 1)$ \\
HPV51 & 165 & $(9 \cdot 3)$ \\
HPV52 & 410 & $(23 \cdot 0)$ \\
HPV53 & 193 & $(10 \cdot 8)$ \\
HPV56 & 56 & $(3 \cdot 1)$ \\
HPV58 & 302 & $(16 \cdot 9)$ \\
HPV59 & 48 & $(4 \cdot 9)$ \\
HPV66 & 88 & $(7 \cdot 0)$ \\
HPV68 & 124 & \\
\hline \hline
\end{tabular}

HPV, Human papillomavirus.

Percentages for individuals co-infected by $\geqslant 2$ high-risk HPV strains were calculated respectively.

status, the co-infection rates of the three most dominant high-risk HPVs were calculated. As shown in Table 2 , only $60 \cdot 0 \%$ of cases positive for HPV genotype 52 were mono-infections, and the rates of HPV52 co-infection with HPV16, 18, 31, 33, 35, 39, $45,51,53,56,58,59,66$, and 68 were $5 \cdot 4 \%, 4 \cdot 9 \%$, $4 \cdot 4 \%, 4 \cdot 1 \%, 0 \cdot 5 \%, 6 \cdot 8 \%, 1 \cdot 0 \%, 4 \cdot 4 \%, 6 \cdot 6 \%, 2 \cdot 7 \%$, $8 \cdot 3 \%, 1 \cdot 7 \%, 2 \cdot 7 \%$, and $4 \cdot 4 \%$, respectively. Of HPV16 cases, only $67 \cdot 7 \%$ were mono-infections, and the rates of HPV16 co-infection with HPV18, 31, 33, 35, $39,45,51,52,53,56,58,59,66$, and 68 were $3 \cdot 8 \%$,
Table 2. Characteristics of HPV co-infection

\begin{tabular}{lcccc}
\hline \hline & $\begin{array}{l}\text { HPV52 } \\
(N=410)\end{array}$ & $\begin{array}{l}\text { HPV16 } \\
(N=316) \\
n(\%)\end{array}$ & $\begin{array}{l}\text { HPV58 } \\
(N=302)\end{array}$ & $n(\%)$ \\
Genotype & $n(\%)$ & & $P$ \\
\hline HPV16 & $22(5 \cdot 4)$ & $214(67 \cdot 7)$ & $21(7 \cdot 0)$ & $0 \cdot 4272$ \\
HPV18 & $20(4 \cdot 9)$ & $12(3 \cdot 8)$ & $11(3 \cdot 6)$ & $0 \cdot 6603$ \\
HPV31 & $18(4 \cdot 4)$ & $15(4 \cdot 7)$ & $14(4 \cdot 6)$ & $0 \cdot 9704$ \\
HPV33 & $17(4 \cdot 1)$ & $12(3 \cdot 8)$ & $39(12 \cdot 9)$ & $<\mathbf{0 \cdot 0 0 1}$ \\
HPV35 & $2(0 \cdot 5)$ & $0(0)$ & $5(1 \cdot 6)$ & $1 \cdot 0000$ \\
HPV39 & $28(6 \cdot 8)$ & $13(4 \cdot 1)$ & $19(6 \cdot 3)$ & $0 \cdot 2780$ \\
HPV45 & $4(1 \cdot 0)$ & $2(0 \cdot 6)$ & $4(1 \cdot 3)$ & $1 \cdot 0000$ \\
HPV51 & $18(4 \cdot 4)$ & $16(5 \cdot 1)$ & $12(4 \cdot 0)$ & $0 \cdot 8025$ \\
HPV52 & $245(60 \cdot 0)$ & $22(7 \cdot 0)$ & $34(11 \cdot 3)$ & $\mathbf{0 \cdot 0 4 2 5}$ \\
HPV53 & $27(6 \cdot 6)$ & $10(3 \cdot 2)$ & $14(4 \cdot 6)$ & $0 \cdot 1038$ \\
HPV56 & $11(2 \cdot 7)$ & $5(1 \cdot 6)$ & $7(2 \cdot 3)$ & $0 \cdot 6065$ \\
HPV58 & $34(8 \cdot 3)$ & $22(7 \cdot 0)$ & $162(53 \cdot 6)$ & $0 \cdot 3009$ \\
HPV59 & $7(1 \cdot 7)$ & $7(2 \cdot 2)$ & $8(2 \cdot 6)$ & $0 \cdot 6873$ \\
HPV66 & $11(2 \cdot 7)$ & $9(2 \cdot 8)$ & $12(4 \cdot 0)$ & $0 \cdot 5857$ \\
HPV68 & $18(4 \cdot 4)$ & $5(1 \cdot 6)$ & $6(2 \cdot 0)$ & $\mathbf{0} \cdot \mathbf{0 4 4 6}$ \\
& & & & \\
\hline
\end{tabular}

HPV, Human papillomavirus.

Differences in co-infection were examined by $c^{2}$ test or Fisher's exact probability test according to the characteristics of the data distribution.

$4 \cdot 7 \%, \quad 3 \cdot 8 \%, 0 \%, 4 \cdot 1 \%, 0 \cdot 6 \%, 5 \cdot 1 \%, 7 \cdot 0 \%, \quad 3 \cdot 2 \%$, $1 \cdot 6 \%, 7 \cdot 0 \%, 2 \cdot 2 \%, 2 \cdot 8 \%$, and $1 \cdot 6 \%$, respectively (Table 2). Only $53 \cdot 6 \%$ of cases positive for HPV58 were mono-infections, and the rates of HPV58 co-infected with HPV16, 18, 31, 33, 35, 39, 45, 51, $52,53,56,59,66$, and 68 were $7 \cdot 0 \%, 3 \cdot 6 \%, 4 \cdot 6 \%$, $12 \cdot 9 \%, 1 \cdot 6 \%, 6 \cdot 3 \%, 1 \cdot 3 \%, 4 \cdot 0 \%, 11 \cdot 3 \%, 4 \cdot 6 \%, 2 \cdot 3 \%$, $2 \cdot 6 \%, 4.0 \%$, and $2 \cdot 0 \%$, respectively (Table 2 ). The greatest number of high-risk HPV strains detected in a single subject was seven; a 55-year-old woman was 
simultaneously co-infected with HPV $31,35,52,53$, 39, 66, and 68. HPV58/HPV33 and HPV58/HPV52 showed significantly higher co-infection rates, suggesting that HPV58 is the most frequent co-infection highrisk HPV in this population (Table 2). HPV58/HPV68 and HPV16/HPV68 showed significantly lower co-infection rates, suggesting that HPV68 may be the rarest co-infection strain in our population (Table 2).

\section{High-risk HPV genotype distribution differs regionally}

To determine the differences in HPV genotype distributions, we reviewed recent representative reports from the National Center for Biotechnology Information. In high-risk HPV-infected subjects, the three most dominant high-risk HPV strains (HPV16, $52,58)$ were the same in six studies performed in Chinese populations, although the ratios varied between studies, while HPV53 was among the three most dominant strains instead of HPV52 in one study performed in Harbin (Table 3). However, the order from high to low varied widely. In the present study, the order was HPV52, HPV16, and HPV58 in order of decreasing prevalence (Table 3). Furthermore, other genotype distributions also differed regionally in China (Table 3). Clifford et al. [26] reported a distribution tendency worldwide, with HPV16, 31, and 51 representing the three most dominant HPV genotypes (Table 3). Of these, only HPV16 is among the three most dominant strains in China. In the general population included in our study, the prevalence rates of HPV52, 16, and 58 were $3.9 \%, 3.0 \%$, and $2.9 \%$, respectively (Table 3 ). Data from India, Northern Ireland and the USA varied widely, except for HPV16 which remained a dominant strain worldwide [27-30]. The genotype distribution differed regionally worldwide.

\section{DISCUSSION}

Cervical cancer is estimated to affect about half a million women each year, of whom $80 \%$ live in developing countries [31]. Virtually all cases of cervical cancer are caused by HPV infection [1-3]. Well-organized programmes of regular gynaecological screening and treatment of precancerous lesions have been very effective for preventing squamous cervical cancer but have had less impact on adenocarcinoma [31]. Fortunately, HPV vaccines were licensed for use in several countries in 2006, and adenocarcinoma is now expected to be controlled by HPV vaccines [32]. However, currently available HPV vaccines are not region-specific. That is, current vaccination strategies do not adequately take into consideration the distribution and prevalence characteristics of HPV genotypes. Both the quadrivalent and bivalent vaccines protect against HPV types 16 and 18 , which cause $70 \%$ of cervical cancers; the quadrivalent vaccine also protects against HPV types 6 and 11, which cause $90 \%$ of genital wart cases [33]. Although it is difficult to map the HPV genotype distribution and prevalence, large amounts of data indicate that HPV genotype distribution varies largely across regions [21-30]. Thus, regional HPV surveillance is essential for effective vaccination and HPV-related cancer control and prevention. In this study, we found that $17 \cdot 3 \%$ of outpatients undergoing cervical screening were infected with high-risk HPVs. Of these subjects, 24.5\% were infected with $\geqslant 2$ HPV strains, and the average age of subjects infected with $\geqslant 3$ HPV strains was significantly younger than those of other groups. The three most dominant HPV genotypes prevalent in our population were HPV52, HPV16, and HPV58. Of the three most dominant HPV strains, only $60.0 \%$ of subjects infected with HPV52, $67 \cdot 7 \%$ of subjects infected with HPV16, and $53.6 \%$ of subjects infected with HPV58 were mono-infections, with the remainder being co-infected with other high-risk HPVs. HPV58/ HPV33 (12.9\%) and HPV58/HPV52 (11.3\%) showed significantly higher co-infection rates than other combinations. HPV genotype distribution varied markedly between domestic and international regions, while HPV16 remained among the three most dominant strains worldwide. Our data emphasize the complexity of HPV genotype distribution and the importance of local HPV surveys for vaccination strategies.

The prevalence rates of high-risk HPVs according to age are somewhat confusing, with $20 \cdot 6 \%, 14.9 \%$, $15.9 \%, 17 \cdot 4 \%$, and $21 \cdot 2 \%$ of subjects in the $\leqslant 24$, $25-34,35-44,45-55$, and $>55$ years age groups, respectively. The majority of women who attended for cervical screening were aged 25-34 years. In contrast, women with cervical diseases who visited our department spontaneously, belonged primarily to the other age groups. This might explain why the lowest HPV positivity rate was seen in the population aged 25-34 years. Although we could not give a scientific explanation for the age-specific prevalence rates, our data are similar to the distribution tendencies in central and South America and Europe reported in a metaanalysis [30]. Thus, our data may represent the real 
Table 3. Summary of related reports

\begin{tabular}{|c|c|c|c|c|c|c|c|c|c|c|c|c|c|c|c|c|c|c|}
\hline \multirow[b]{2}{*}{ Source } & \multicolumn{15}{|c|}{ High-risk HPV genotypes } & \multirow[b]{2}{*}{ Region } & \multirow[b]{2}{*}{ Population } & \multirow[b]{2}{*}{ Number } \\
\hline & 16 & 18 & 31 & 33 & 35 & 39 & 45 & 51 & 52 & 53 & 56 & 58 & 59 & 66 & 68 & & & \\
\hline \multicolumn{19}{|l|}{$\begin{array}{l}\text { Ratios in high-risk } \\
\text { HPV-infected subjects }\end{array}$} \\
\hline Our data & $17 \cdot 7$ & $6 \cdot 3$ & $7 \cdot 5$ & $10 \cdot 4$ & $2 \cdot 2$ & $9 \cdot 3$ & $2 \cdot 1$ & $9 \cdot 3$ & $23 \cdot 0$ & $10 \cdot 8$ & $3 \cdot 1$ & $16 \cdot 9$ & $2 \cdot 7$ & $4 \cdot 9$ & $7 \cdot 0$ & Shanghai & Outpatient & 1782 \\
\hline Sun et al. [21] & $30 \cdot 3$ & $5 \cdot 1$ & $4 \cdot 9$ & $7 \cdot 5$ & $2 \cdot 2$ & $4 \cdot 9$ & $1 \cdot 3$ & $2 \cdot 1$ & $7 \cdot 0$ & $9 \cdot 1$ & $5 \cdot 2$ & $12 \cdot 6$ & $2 \cdot 8$ & $4 \cdot 4$ & $8 \cdot 0$ & Harbin & Outpatient & 988 \\
\hline Hong et al. [22] & $11 \cdot 8$ & $5 \cdot 1$ & $5 \cdot 1$ & $10 \cdot 1$ & $2 \cdot 8$ & $3 \cdot 9$ & $1 \cdot 7$ & $2 \cdot 8$ & $17 \cdot 4$ & $4 \cdot 5$ & $6 \cdot 2$ & $14 \cdot 6$ & $2 \cdot 2$ & $2 \cdot 8$ & $6 \cdot 7$ & Ningbo & Community & 178 \\
\hline Zhi et al. [23] & $22 \cdot 2$ & $0 \cdot 8$ & $4 \cdot 8$ & $6 \cdot 3$ & $3 \cdot 4$ & $0 \cdot 9$ & $1 \cdot 2$ & $2 \cdot 7$ & $12 \cdot 1$ & $4 \cdot 1$ & $8 \cdot 6$ & $13 \cdot 5$ & $4 \cdot 0$ & $7 \cdot 6$ & $5 \cdot 2$ & Zhengzhou & Outpatient & 3690 \\
\hline Xue et al. [24] & $26 \cdot 2$ & $7 \cdot 5$ & $6 \cdot 5$ & $7 \cdot 6$ & $1 \cdot 5$ & $3 \cdot 7$ & $1 \cdot 5$ & $1 \cdot 0$ & $19 \cdot 4$ & $13 \cdot 3$ & $1 \cdot 8$ & $13 \cdot 8$ & $2 \cdot 4$ & $5 \cdot 0$ & $6 \cdot 3$ & Liaoning & Physical examination & 598 \\
\hline Bi et al. [25] & $21 \cdot 4$ & $4 \cdot 1$ & $3 \cdot 9$ & $3 \cdot 4$ & $3 \cdot 7$ & $2 \cdot 0$ & $1 \cdot 5$ & $5 \cdot 2$ & $11 \cdot 9$ & $8 \cdot 7$ & $5 \cdot 5$ & $10 \cdot 5$ & $4 \cdot 2$ & $6 \cdot 2$ & $5 \cdot 4$ & Qingdao & Outpatient & 1768 \\
\hline Clifford et al. [26] & $26 \cdot 6$ & $8 \cdot 6$ & $11 \cdot 7$ & $7 \cdot 6$ & $5 \cdot 9$ & $7 \cdot 8$ & $4 \cdot 9$ & $10 \cdot 9$ & $8 \cdot 8$ & $10 \cdot 1$ & $9 \cdot 7$ & $8 \cdot 5$ & $6 \cdot 0$ & $8 \cdot 5$ & - & Worldwide & $\begin{array}{l}\text { Low-grade squamous } \\
\text { intraepithelial lesions }\end{array}$ & 5910 \\
\hline \multicolumn{19}{|c|}{ Ratios in general population } \\
\hline Our data & $3 \cdot 0$ & $1 \cdot 1$ & $1 \cdot 3$ & $1 \cdot 8$ & $0 \cdot 4$ & $1 \cdot 6$ & $0 \cdot 4$ & $1 \cdot 6$ & $3 \cdot 9$ & $1 \cdot 8$ & $0 \cdot 5$ & $2 \cdot 9$ & $0 \cdot 5$ & $0 \cdot 8$ & $1 \cdot 2$ & Shanghai & Outpatient & 10501 \\
\hline Bhatla et al. [27] & $3 \cdot 2$ & $0 \cdot 7$ & $0 \cdot 5$ & $0 \cdot 6$ & $0 \cdot 5$ & $0 \cdot 5$ & $0 \cdot 2$ & $0 \cdot 3$ & $0 \cdot 7$ & - & $0 \cdot 7$ & $0 \cdot 3$ & $0 \cdot 5$ & - & $0 \cdot 0$ & India & Heath women & $1048-3061$ \\
\hline Anderson et al. [28] & $3 \cdot 2$ & $1 \cdot 2$ & $1 \cdot 8$ & $1 \cdot 2$ & $0 \cdot 5$ & $1 \cdot 2$ & $1 \cdot 0$ & $1 \cdot 6$ & $1 \cdot 5$ & - & $1 \cdot 0$ & $0 \cdot 8$ & $1 \cdot 6$ & $1 \cdot 3$ & $0 \cdot 5$ & $\begin{array}{l}\text { Northern } \\
\text { Ireland }\end{array}$ & Normal cervical cytology & 5068 \\
\hline Wheeler et al. [29] & $7 \cdot 4$ & $2 \cdot 3$ & $2 \cdot 9$ & $0 \cdot 9$ & $1 \cdot 0$ & $3 \cdot 3$ & $2 \cdot 2$ & $3 \cdot 0$ & $2 \cdot 9$ & $4 \cdot 9$ & $2 \cdot 4$ & $2 \cdot 5$ & $2 \cdot 1$ & $2 \cdot 3$ & $2 \cdot 1$ & United States & Cervical screening & 4007 \\
\hline de Sanjosé et al. [30] & $2 \cdot 5$ & $0 \cdot 9$ & $0 \cdot 7$ & - & - & - & - & - & $0 \cdot 6$ & - & - & $0 \cdot 6$ & - & - & - & Worldwide & Normal cervical cytology & $\sim 15764$ \\
\hline
\end{tabular}

HPV, Human papillomavirus.

Data are presented as percentage (\%). The three most dominant genotypes are shown in bold. 
age-specific distribution characteristics that may be determined by bearing, female hormone dynamics, sex, and age of the host. On the other hand, our data also indicate that the average ages of subjects infected by one, two, and $\geqslant 3 \mathrm{HPV}$ strains were $39 \cdot 5 \pm 12 \cdot 4,40 \cdot 2 \pm$ 13.6 , and $36.6 \pm 15.8$ years, respectively. The average age of subjects infected by $\geqslant 3$ HPV strains was significantly lower than that of the other two groups. As HPV is a sexually transmitted disease, the relatively high frequency of sexual intercourse and greater number of sexual partners might explain why multiple infections are more frequent in younger women. With regard to multiple infections, few studies have investigated the natural history of multiple HPV infection, how and when these HPV strains infect the same host, the impact of multiple infections on cervical cancer, and any tendency for an affinity between HPVs in co-infecting the same host. These questions are also important for HPV virological studies. However, these issues are outside the scope of this epidemiological study.

Further comparative analysis showed that HPV16, 52 , and 58 are the three most dominant high-risk HPV strains in five Chinese reports, although the order of prevalence rates from high to low varied widely and other genotype distributions also differed regionally in China [21-25]. Other data from India, Northern Ireland, the USA and integrated data varied except for HPV16, which remained a dominant strain worldwide [26-30]. Although all data showed that HPV16 is a dominant strain worldwide, the highest percentage of HPV16 infection was only $26 \cdot 2 \%$ in high-risk HPV-infected subjects, and therefore the genotype distribution differed regionally worldwide. Again, our report emphasizes the complexity of HPV genotype distribution and the importance of local HPV surveys.

In this study, we detected only 15 high-risk HPV strains (HPV16, 18, 31, 33, 35, 39, 45, 51, 52, 53, 56, 58, 59, 66, 68) and six low-risk HPV strains (HPV6, 11, 42, 43, 44, CP8304), and we could not determine the distribution characteristics of other HPVs in women living in Shanghai. HPV genotype distribution is known to differ with pathological grade. We are in the process of analysing these data regarding HPV infection of different pathological grades and will report the results in the near future.

\section{ACKNOWLEDGEMENTS}

This work was supported by a grant as a Leading Academic Discipline Project of the Shanghai 7th People's Hospital (xk2013-01).

\section{DECLARATION OF INTEREST}

None.

\section{REFERENCES}

1. Moore PS, Chang Y. Why do viruses cause cancer? Highlights of the first century of human tumour virology. Nature Reviews Cancer 2010; 10: 878-889.

2. Bian T, et al. Human papillomavirus type 16 L1E7 chimeric capsomeres have prophylactic and therapeutic efficacy against papillomavirus in mice. Molecular Cancer Therapeutics 2008; 7: 1329-1335.

3. Dillner J. Prevention of human papillomavirus-associated cancers. Seminars in Oncology 2015; 42: 272-283.

4. Winer RL, et al. Condom use and the risk of genital human papillomavirus infection in young women. New England Journal of Medcine 2006; 354: 2645-2654.

5. Chaturvedi AK, et al. Human papillomavirus and rising oropharyngeal cancer incidence in the United States. Journal of Clinical Oncology 2011; 29: 4294-42301.

6. Saraiya M, et al. US assessment of HPV types in cancers: implications for current and 9-valent HPV vaccines. Journal of the National Cancer Institute 2015; 107: djv086.

7. Globocan. Cervical cancer estimated incidence, mortality, and prevalence worldwide in 2012 (http://globocan. iarc.fr/old/FactSheets/cancers/cervix-new.asp). Accessed 7 August 2015.

8. Steenbergen RD, et al. Clinical implications of (epi)genetic changes in HPV-induced cervical precancerous lesions. Nature Reviews Cancer 2014; 14: 395-405.

9. Johansson C, Schwartz S. Regulation of human papillomavirus gene expression by splicing and polyadenylation. Nature Reviews Microbiology 2013; 11: 239-251.

10. Abbate EA, Voitenleitner C, Botchan MR. Structure of the papillomavirus DNA-tethering complex E2: Brd4 and a peptide that ablates HPV chromosomal association. Molecular Cell 2006; 24: 877-889.

11. Ghittoni R, et al. Role of human papillomaviruses in carcinogenesis. Ecancermedicalscience 2015; 9: 526.

12. Muñoz N, et al. Epidemiologic classification of human papillomavirus types associated with cervical cancer. New England Journal of Medicine 2003; 348: 518-527.

13. Szarewski A, et al. Comparison of seven tests for highgrade cervical intraepithelial neoplasia in women with abnormal smears: the Predictors 2 study. Journal of Clinical Microbiology 2012; 50: 1867-1873.

14. Eide ML, Debaque H. HPV detection methods and genotyping techniques in screening for cervical cancer. Annales De Pathologie 2012; 32: e15-23, 401-409.

15. Lacey CJ, Lowndes CM, Shah KV. Chapter 4: Burden and management of non-cancerous HPV-related conditions: HPV-6/11 disease. Vaccine 2006; 24: S35-41.

16. Elrefaey S, et al. HPV in oropharyngeal cancer: the basics to know in clinical practice. Acta Otorinorhinolaryngologica Italica 2014; 34: 299-309.

17. Kreimer AR, et al. Human papillomavirus types in head and neck squamous cell carcinomas worldwide: a 
systematic review. Cancer Epidemiology, Biomarkers \& Prevention 2005; 14: 467-75.

18. Muñoz N, et al. Chapter 1: HPV in the etiology of human cancer. Vaccine 2006; 24 Suppl 3: S3/1-10.

19. Barcelos AC, et al. Atypical squamous cells of undetermined significance: Bethesda classification and association with human papillomavirus. Infectious Diseases in Obstetrics and Gynecology 2011; 2011: 904674.

20. American Society for Colposcopy and Cervical Pathology. HPV genotyping clinical update (www. asccp.org/pdfs/consensus/clinical_update_20090408.pdf). Accessed 7 August 2015.

21. Sun B, et al. Prevalence and genotype distribution of human papillomavirus infection in Harbin, Northeast China. Archives of Virology 2014; 159: 1027-1032.

22. Hong $\mathbf{H}$, et al. Prevalence and genotype distribution of HPV infection among women in Ningbo, China. International Journal of Gynecology \& Obstetrics 2015; 131: 96-99.

23. Zhi YF, et al. Prevalence and genotype distribution of human papillomavirus in women in the Henan Province. Genetics and Molecular Research 2015; 14: 5452-5461.

24. Xue H, et al. Prevalence and genotype distribution of human papillomavirus infection in asymptomatic women in Liaoning province, China. Journal of Medical Virology 2015; 87: 1248-1253.

25. Bi Q, et al. Human papillomavirus prevalence and genotypes distribution among female outpatients in Qingdao, East China. Journal of Medical Virology. Published online: 9 June 2015. doi:10.1002/jmv.24281.

26. Clifford GM, et al. Human papillomavirus genotype distribution in low-grade cervical lesions: comparison by geographic region and with cervical cancer. Cancer Epidemiology, Biomarkers \& Prevention 2005; 14: 1157-1164.

27. Bhatla $\mathbf{N}$, et al. A meta-analysis of human papillomavirus type-distribution in women from South Asia: implications for vaccination. Vaccine 2008; 26: 2811-7.

28. Anderson L, et al. Prevalence of human papillomavirus in women attending cervical screening in the UK and Ireland: new data from Northern Ireland and a systematic review and meta-analysis. Journal of Medical Virology 2013; 85: 295-308.

29. Wheeler CM, et al. Human papillomavirus genotype distributions: implications for vaccination and cancer screening in the United States. Journal of the National Cancer Institute 2009; 101: 475-487.

30. de Sanjosé S, et al. Worldwide prevalence and genotype distribution of cervical human papillomavirus DNA in women with normal cytology: a meta-analysis. Lancet Infectious Diseases 2007; 7: 453-459.

31. Chew GK, et al. Human papillomavirus 16 infection in adenocarcinoma of the cervix. British Journal of Cancer 2005; 93: 1301-1304.

32. Stokley S, et al. Human papillomavirus vaccination coverage among adolescents, 2007-2013, and postlicensure vaccine safety monitoring, 2006-2014-United States. Morbidity and Mortality Weekly Report 2014; 63: $620-4$.

33. CDC. FDA licensure of bivalent human papillomavirus vaccine (HPV2, Cervarix) for use in females and updated HPV vaccination recommendations from the Advisory Committee on Immunization Practices (ACIP). Morbidity and Mortality Weekly Report 2010; 59: 626-629. 